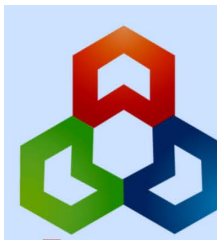

FRONTIER

RESEARCH JOURNALS

(RESEARCH ARTICLE)

\title{
Histological and body weight effects of Datura innoxia seeds and leaves extracts in rats
}

\author{
Ali A. Eltayeib * and Siddige A. N. T. Matter \\ Department of Chemistry, Faculty of Science, Kordofan University, Sudan.
}

International Journal of Frontiers in Life Science Research, 2021, 01(01), 018-027

Publication history: Received on 04 March 2021; revised on 10 April 2021; accepted on 14 April 2021

Article DOI: https://doi.org/10.53294/ijflsr.2021.1.1.0032

\begin{abstract}
The study aimed to determine the chemical compounds in aqueous and methanolic extracts of Datura innoxia seeds and leaves and to evaluate their toxic effects on experimental rats. Seeds and leaves were collected from El-Obied, North Kordofan State, Sudan, in October, 2016. The aqueous and methanol extracts were carried out by using maceration method and soxhlet apparatus respectively. Sixty five male Albino Wistar rats, three months old and with an average body weight ranged 110-120 g, were randomly divided into thirteen groups, consisting of five rats in each group. Group1 served as control and fed with normal rats' food and water for thirty days. Groups 2, 6 and 10 administered aqueous seeds extract, Groups 4, 8 and 12 received methanol seeds extract, Groups 3, 7 and 11 received aqueous leaves extracts, Groups 5, 9 and 13 received methanol leaves extract, all the groups received the same type of extract were administered 40,60 and $80 \mathrm{mg} / \mathrm{kg}$ body weight respectively. The extracts administered to the rats intra gastrically using cathodal tube daily for thirty days. The effects of oral administration of leaves and seeds extracts to60 healthy rats over 30 days were evaluated by histological studies and body weight changes. The analysis by gas chromatography-mass spectrometry (GC-MS) of aqueous and methanolic extracts revealed the presence of alkaloids (Scopolamine, atropine and Hyoscyamine), fatty acids, esters, amides, amino acids, ketones, coummarins, terpinoids, phenols, alcohols and hydrocarbons compounds. The histological results showed that administration of extracts caused pathologic changes in the organs studied. The treated Groups had lower $(\mathrm{p} \leq 0.05)$ body weight gains than control Group. The study concluded that the toxicity of seeds and leaves (methanolic and aqueous) extracts are nearly have the same toxic effects on rats due to their same active ingredients (alkaloids) and the oral administration of the extracts was found to be safe up to $40 \mathrm{mg} / \mathrm{kg}$.
\end{abstract}

Keywords: Datura innoxia; Seeds and leaves; Chemical composition; Histological studies; Body weight.

\section{Introduction}

From the ancient time the knowledge about plants and their medicinal values were existing. All these knowledge have been transmitted from generations to generations but a little is known on their toxicity and about a suitable dose. Medicinal plants are traditionally used for the treatment of human infections and have made large contribution to human health [1]. According to World Health Organization (WHO), medicinal plants would be the best source to obtain a variety of drugs [2]. Those plants are widely used by all sections of community, whether directly as folk remedies or the medicaments of the different indigenous systems as well as in modern medicine system [3]. D. innoxia is native to the tropical and subtropical Americas, from south western USA and Mexico, through Central America to northern and western South America, to Peru, and east to Bolivia and Paraguay. In the USA it is native only to Texas [4], butis also recorded in the southwestern states, Arkansas and in the northeast USA as far as Ontario and Quebec, Canada [5]. D. innoxia has been listed as a declared weed in South Africa which is prohibited and must be controlled [6]. Daturainnoxia is one of the medicinal plants used by traditional healers in Sudan for the treatment of various ailments. Datura species,

\footnotetext{
* Corresponding author: Ali A. Eltayeib

Department of Chemistry, Faculty of Science, Kordofan University, Sudan.
} 
particularly the seeds have been used in shamanistic rituals as a path to enlightenment since ancient times [7]. All parts of the plant are anodyne, antispasmodic, hallucinogenic, hypnotic and narcotic [8]. It has been used in the past as a painkiller and also in the treatment of insanity, fevers with catarrh, diarrhoea and skin diseases. The plant contains several alkaloids, the most active of which is scopolamine [9]. This is a potent cholinergic-blocking hallucinogen, which has been used to calm schizoid patients [10].

\section{Material and methods}

\subsection{Plant Materials (Seeds and Leaves)}

D. innoxia seeds and leaves were collected from Elobied, North Kordofan state, Sudan in October, 2016. The plant was authenticated by a plant taxonomist at the Department of Botany Faculty of Science University of Kordofan to be Daturainnoxia. The plant leaves and seeds were cleaned, shade-dried and grinded by a mechanical grinder.

\subsection{Animals (rats)}

Sixty five male Wister rats, three months old and with an average body weight ranged (110-120g), were used in the present study. The rats were clinically healthy and housed within the premises of the Faculty of Science and Technology, Sudan University, Khartoum. Animal housed under standard husbandry conditions $\left(30^{\circ} \mathrm{C} \pm 2{ }^{\circ} \mathrm{C}, 60-70 \%\right.$ relative humidity and 12 hour day-night cycle) and fed on the rat diet (flour 55.6\%, meat 35\%, edible oil 7.5\%, sodium chloride $1.2 \%$ and vitamins and minerals $0.7 \%$ ) and water provider. Animal experiments were designed and conducted in accordance with the guidelines of institutional animal ethical committee.

\subsection{Methods}

Extraction by Petroleum ether and methanol: The extraction was carried out according to method described by [11]: $600 \mathrm{~g}$ of each coarsely powdered sample (seeds and leaves) was successively extracted with 1200 ml of petroleum ether and $1200 \mathrm{ml}$ of methanol using soxhlet extractor apparatus. Extraction was carried out for about four hours for petroleum ether and eight hours for methanol till the color of solvents at the last siphoning time returned colorless. Solvents were evaporated under reduced pressure using rotary evaporator apparatus. Finally the extracts allowed to air till complete dryness.

\subsection{Extraction by water}

For aqueous extract, $600 \mathrm{~g}$ of each coarsely powdered sample (seeds and leaves) was extracted with $3000 \mathrm{ml}$ of distilled water and heat to $70^{\circ} \mathrm{C}$ for three hours and filtered through what man paper No. 0.1 and dried further by freeze drier.

\subsection{Preparation of stock solutions}

The preparation of stock solution was done by dissolving $5 \mathrm{~g}$ of each extract in $12.5 \mathrm{ml}$ of $99.9 \%$ ethyl alcohol and completed to $250 \mathrm{ml}$ with distilled water and then $2 \mathrm{ml}$; $3 \mathrm{ml}$ and $4 \mathrm{ml}$ (40 mg, $60 \mathrm{mg}$ and $80 \mathrm{mg}$ ) were taken from the stock solution and used as doses for rats orally.

\subsection{Experimental Design}

A total of sixty five male Wistar albino rats divided into thirteen Groups containing five each. Group 1served as control and fed with normal rat food and water for thirty days. Groups 4, 8 and 12 received methanol seeds extract with dose of 40,60,80mg/kg body weight/day respectively and Groups 5, 9 and 13 received methanol leaves extract with dose of 40,60 , and $80 \mathrm{mg} / \mathrm{kg}$ body weight /day respectively. Groups 2, 6 and 10 received aqueous seeds extract with dose of 40,60, $80 \mathrm{mg} / \mathrm{kg}$ body weight/day respectively and Groups 3, 7 and 11 received aqueous leaves extract with dose of 40, 60 , and $80 \mathrm{mg} / \mathrm{kg}$ body weight / day respectively. The extract administered to the rats intra gastricallyusing cathodal tube for thirty days. The method was prepared according to the Standard Method of Organization for Economic Cooperation and Development [12]. The daily feed intake was monitored in the rats until termination of the experiment.

\subsection{Histopathological study}

Necropsy was conducted to identify gross lesion. The specimens of liver, kidney and brain were collected after slaughtering of rats and immediately fixed in 10\% neutral buffered formalin, embedded in paraffin wax, sectioned at 5 $\mathrm{cm}$ and stained routinely with hematoxylin and eosin using Harris's heamalun [13]. 


\subsection{GC-MS analysis conditions}

The chemical composition analysis of the samples were carried out by using GC/MS technique model -TQ8040 from Japan with capillary column (RTX-5MS), column oven temperature $80^{\circ} \mathrm{C}$, injection temp. $250{ }^{\circ} \mathrm{C}$, the sample was injected by using split mode, pressure $122 \mathrm{kpa}$, total flow $50 \mathrm{ml} / \mathrm{min}$, column flow $1.80 \mathrm{ml} / \mathrm{min}$, purge flow $6 \mathrm{ml} / \mathrm{min}$, split ratio -1 , helium as the carrier gas, ion source temp. $200{ }^{\circ} \mathrm{C}$, interface temp. $250{ }^{\circ} \mathrm{C}$, solvent cut time $2.5 \mathrm{~min}$, detector gain +0.30 $\mathrm{kv}$, threshold 1000, start time 3min, end time $21.00 \mathrm{~min}$, acquisition Mode Q3Scan, start m/z 25, end m/z 400, the oven temp. program start from $80^{\circ} \mathrm{C}$ with rate $15^{\circ} \mathrm{C} / \mathrm{min}$ to $200^{\circ} \mathrm{C}$ with 1 min hold time to $260{ }^{\circ} \mathrm{C}$ with rate $10{ }^{\circ} \mathrm{C} / \mathrm{min}$ with $1 \mathrm{~min}$ hold time to $280^{\circ} \mathrm{C}$ as final temperature with 2 min hold time.

\section{Results and discussion}

\subsection{Chemical compounds of methanol extract of Datura innoxia seeds}

The study of chemical composition of methanol extract of Datura innoxia seeds by GC-MS analysis showed the presence of 20 compounds. The chemical compounds with their retention time (RT), chemical formula and molecular weight are presented in Table 1.

Table 1 Chemical compounds of methanol extract of Datura innoxia seeds

\begin{tabular}{|c|c|c|c|c|}
\hline Compound name & Formula & M. wt. & Base ions & RT \\
\hline Scopolamine & $\mathrm{C}_{17} \mathrm{H}_{21} \mathrm{NO}_{4}$ & 303 & $108-138$ & 15.48 \\
\hline Aziridine,1-methyl & $\mathrm{C}_{3} \mathrm{H}_{7} \mathrm{~N}$ & 57 & $42-55$ & 6.08 \\
\hline Tropinone & $\mathrm{C}_{8} \mathrm{H}_{13} \mathrm{NO}$ & 139 & $42-96$ & 15.48 \\
\hline 3-Tropanone & $\mathrm{C}_{8} \mathrm{H}_{13} \mathrm{NO}$ & 139 & $96-82$ & 4.93 \\
\hline Propanamide & $\mathrm{C}_{3} \mathrm{H}_{7} \mathrm{NO}$ & 73 & $44-73$ & 3.56 \\
\hline Methyl vinyl carbinol & $\mathrm{C}_{4} \mathrm{H}_{8} \mathrm{O}$ & 72 & $43-57$ & 3.56 \\
\hline $\begin{array}{l}\text { Pseudoecgoninemethyl } \\
\text { ester }\end{array}$ & $\mathrm{C}_{10} \mathrm{H}_{17} \mathrm{NO}_{3}$ & 199 & $96-199$ & 4.93 \\
\hline Atropine & $\mathrm{C}_{17} \mathrm{H}_{23} \mathrm{NO}_{3}$ & 289 & $124-82$ & 14.38 \\
\hline Apoatropin & $\mathrm{C}_{17} \mathrm{H}_{21} \mathrm{NO}_{2}$ & 271 & $124-271$ & 14.38 \\
\hline Hyoscyamine & $\mathrm{C}_{17} \mathrm{H}_{23} \mathrm{NO}_{3}$ & 289 & $124-289$ & 14.38 \\
\hline Tropacocaine & $\mathrm{C}_{15} \mathrm{H} 1{ }_{9} \mathrm{NO}_{2}$ & 245 & $124-82$ & 14.38 \\
\hline $\begin{array}{l}\text { 8-Azabicyclo }\{3,2,1 \text { octane- } \\
\text { 3,6-diol,acetate (ester) }\end{array}$ & $\mathrm{C}_{12} \mathrm{H}_{19} \mathrm{NO}_{4}$ & 241 & $94-154$ & 15.48 \\
\hline Tigloidine & $\mathrm{C}_{13} \mathrm{H}_{12} \mathrm{NO}_{2}$ & 223 & $124-82$ & 14.37 \\
\hline Homatropine & $\mathrm{C}_{16} \mathrm{H}_{21} \mathrm{NO}_{3}$ & 275 & $124-275$ & 14.36 \\
\hline Benzonitril,2-amino- & $\mathrm{C}_{7} \mathrm{H}_{6} \mathrm{~N}_{2}$ & 118 & $91-118$ & 7.73 \\
\hline Oleic acid & $\mathrm{C}_{18} \mathrm{H}_{34} \mathrm{O}_{2}$ & 282 & $111-83$ & 7.43 \\
\hline Azelaic acid & $\mathrm{C}_{9} \mathrm{H}_{16} \mathrm{O}_{4}$ & 188 & $11-152$ & 7.43 \\
\hline Acetic acid,bromo- & $\mathrm{C}_{2} \mathrm{H}_{3} \mathrm{BrO}_{2}$ & 138 & $43-94$ & 14.37 \\
\hline $\begin{array}{l}\text { 2-methylpyrazine carboxylic } \\
\text { acid }\end{array}$ & $\mathrm{C}_{6} \mathrm{H}_{6} \mathrm{~N}_{2} \mathrm{O}_{2}$ & 138 & $138-94$ & 15.47 \\
\hline $\begin{array}{l}\text { Benzoic acid, } 3,5 \text {-dichloro- } \\
\text {,8-aza-8- methylbicyclo } \\
\{3,2,1\} \text { oct-3- ylester }\end{array}$ & $\begin{array}{l}\mathrm{C}_{15} \mathrm{H}_{17} \mathrm{Cl}_{2} \mathrm{~N} \\
\mathrm{O}_{2}\end{array}$ & 313 & $124-82$ & 14.38 \\
\hline
\end{tabular}




\subsection{Chemical compounds of methanol extract of Datura innoxia leaves}

The chemical compounds identified in the methanol crude extract of Datura innoxia leaves are listed in Table 2. The analysis by GC-MS revealed 30 compounds.

Table 2 Chemical compounds of methanol crude extract of Datura innoxia leaves

\begin{tabular}{|c|c|c|c|c|}
\hline Compound name & Formula & M. wt. & Base ions & RT \\
\hline Alanine (Amino acid) & $\mathrm{C}_{3} \mathrm{H}_{7} \mathrm{NO}_{2}$ & 89 & $40-44$ & 18.15 \\
\hline 8-Abicycl $\{3,2,1\}$ octane,3- chloro-8- methyl & $\mathrm{C}_{8} \mathrm{H}_{14} \mathrm{C} 1 \mathrm{~N}$ & 159 & $124-44$ & 14.38 \\
\hline Atropine & $\mathrm{C}_{17} \mathrm{H}_{23} \mathrm{NO}_{3}$ & 289 & $124-67$ & 14.38 \\
\hline Tropacocaine & $\mathrm{C}_{15} \mathrm{H}_{19} \mathrm{NO}_{2}$ & 245 & $124-82$ & 14.38 \\
\hline Benzoicacid,3,5-dichloro-,8-aza-8-bicycle $\{3,2,1\}$ oct-3-yl ester & $\mathrm{C}_{15} \mathrm{H} 1 \mathrm{C} 1_{2} \mathrm{NO}_{2}$ & 313 & $124-94$ & 14.38 \\
\hline Acetamide,2-cyano & $\mathrm{C}_{3} \mathrm{H}_{4} \mathrm{~N}_{2} \mathrm{O}$ & 84 & $40-84$ & 8.60 \\
\hline Phosphorodiamidous Fluoride, tetramethyl & $\mathrm{C}_{4} \mathrm{H}_{12} \mathrm{FN}_{2} \mathrm{P}$ & 138 & $94-138$ & 15.47 \\
\hline d1-phenylephrine & $\mathrm{C}_{19} \mathrm{H}_{13} \mathrm{NO}_{2}$ & 167 & $167-148$ & 11.95 \\
\hline 4-Hydroxy-2-methylaceto phenome & $\mathrm{C}_{9} \mathrm{H}_{10} \mathrm{O}_{2}$ & 150 & $150-107$ & 5.950 \\
\hline Phenol,m-tert-butyl- & $\mathrm{C}_{10} \mathrm{H}_{14} \mathrm{O}$ & 150 & $135-77$ & 5.95 \\
\hline 4-H-pyran-4-one,2,3-3,5-dihydroxy-6- methyl & $\mathrm{C}_{6} \mathrm{H}_{8} \mathrm{O}_{4}$ & 144 & $144-72$ & 4.26 \\
\hline 4-Acetophenone,4-methoxy & $\mathrm{C}_{9} \mathrm{H}_{10} \mathrm{O}_{2}$ & 150 & $135-92$ & 5.95 \\
\hline 2-Hydroxy-5-methylaceto phenone & $\mathrm{C}_{9} \mathrm{H}_{10} \mathrm{O}_{2}$ & 150 & $150-135$ & 5.95 \\
\hline Hyoscyamine & $\mathrm{C}_{17} \mathrm{H}_{23} \mathrm{NO}_{3}$ & 289 & $124-82$ & 13.26 \\
\hline Apoatropin & $\mathrm{C}_{17} \mathrm{H}_{21} \mathrm{NO}_{2}$ & 271 & $94-124$ & 14.38 \\
\hline 4-Hydroxy-3-methyl, acetophenone & $\mathrm{C}_{9} \mathrm{H}_{10} \mathrm{O}_{2}$ & 150 & $135-121$ & 5.95 \\
\hline 4-Hydroxy-2-methylaceto phenone & $\mathrm{C}_{9} \mathrm{H}_{10} \mathrm{O}_{2}$ & 150 & $150-77$ & 5.95 \\
\hline 4H-pyran-4-one,2,3-dihydro-3,5-dihydroxy-6-methyl & $\mathrm{C}_{6} \mathrm{H}_{8} \mathrm{O}_{4}$ & 144 & $140-101$ & 4.26 \\
\hline Acetamide,N-(aminocarbonyl) -2-chloroacetylurea & $\mathrm{C}_{3} \mathrm{H}_{5} \mathrm{C} 1 \mathrm{~N}_{2} \mathrm{O}_{2}$ & 136 & $87-136$ & 11.95 \\
\hline Scopolamine & $\mathrm{C}_{17} \mathrm{H}_{21} \mathrm{NO}_{4}$ & 289 & $124-94$ & 14.36 \\
\hline Pyraanone & $\mathrm{C}_{6} \mathrm{H}_{8} \mathrm{O}_{4}$ & 144 & $73-101$ & 4.25 \\
\hline Phenylephrine & $\mathrm{C}_{9} \mathrm{H}_{13} \mathrm{NO}_{2}$ & 167 & $162-146$ & 11.95 \\
\hline L-Glutamine & $\mathrm{C}_{5} \mathrm{H}_{10} \mathrm{NO}_{3}$ & 146 & $41-84$ & 6.22 \\
\hline Aminopyrazine & $\mathrm{C}_{4} \mathrm{H}_{5} \mathrm{~N}_{3}$ & 95 & $43-95$ & 10.95 \\
\hline 3-Methoxy Acetophenone & $\mathrm{C}_{9} \mathrm{H}_{10} \mathrm{O}_{4}$ & 150 & $77-135$ & 5.95 \\
\hline Hyoscyamine & $\mathrm{C}_{17} \mathrm{H}_{23} \mathrm{NO}_{3}$ & 289 & $124-82$ & 13.26 \\
\hline Phenol,3-(1,1-dimethyl) & $\mathrm{C}_{10} \mathrm{H}_{14} \mathrm{O}$ & 150 & $135-150$ & 5.95 \\
\hline 1,2-Propanediamine & $\mathrm{C}_{3} \mathrm{H}_{10} \mathrm{~N}_{2}$ & 74 & $44-71$ & 13.48 \\
\hline Tigloidine & $\mathrm{C}_{13} \mathrm{H}_{21} \mathrm{NO}_{2}$ & 223 & $82-124$ & 14.38 \\
\hline 2-Hexanamine,4-methyl & $\mathrm{C}_{7} \mathrm{H}_{17} \mathrm{~N}$ & 115 & $44-84$ & 8.60 \\
\hline
\end{tabular}

\subsection{Chemical compounds of aqueous crude extract of Datura innoxia seeds}

The chemical compounds identified in aqueous crude extract of Datura innoxia seedsare listed in Table 3 . The aqueous crude extract was analyzed by using GC-MS leading to the identification of 24 different compounds. 
Table 3 Chemical compounds of aqueous crude extract of Datura innoxia seeds

\begin{tabular}{|c|c|c|c|c|}
\hline Compound name & Formula & M. wt. & Base ions & RT \\
\hline 4H-Pyran-4-one, 2,3-dihydro-3,5-dihydroxy-6-methyl & $\mathrm{C}_{6} \mathrm{H}_{8} \mathrm{O}_{4}$ & 144 & 144-101 & 4.25 \\
\hline 2,4-Dihydroxy- dimethyl-3(2H)-furan-3-one & $\mathrm{C}_{6} \mathrm{H}_{8} \mathrm{O}_{4}$ & 144 & $144-101$ & 4.25 \\
\hline Sucrose & $\mathrm{C}_{12} \mathrm{H}_{22} \mathrm{O}_{11}$ & 342 & $163-85$ & 6.82 \\
\hline 3-Nitrobenzl bromide & $\mathrm{C}_{7} \mathrm{H}_{6} \mathrm{BrNO}_{2}$ & 215 & $215-90$ & 7.08 \\
\hline Uric acid & $\mathrm{C}_{5} \mathrm{H}_{4} \mathrm{~N}_{4} \mathrm{O}_{3}$ & 168 & $168-125$ & 8.08 \\
\hline VaniLlic acid & $\mathrm{C}_{8} \mathrm{H}_{8} \mathrm{O}_{4}$ & 168 & $168-125$ & 8.08 \\
\hline Gllacetophenone & $\mathrm{C}_{8} \mathrm{H}_{8} \mathrm{O}_{4}$ & 168 & $168-125$ & 8.08 \\
\hline 3-Hydroxy-4-methoxy benzoic acid & $\mathrm{C}_{8} \mathrm{H}_{8} \mathrm{O}_{4}$ & 168 & $168-125$ & 8.08 \\
\hline 2-Piperidinone, 1-methyl- & $\mathrm{C}_{6} \mathrm{H}_{11} \mathrm{NO}$ & 113 & $113-57$ & 8.18 \\
\hline Limonene oxide trans & $\mathrm{C}_{10} \mathrm{H}_{16} \mathrm{O}$ & 152 & $94-108$ & 15.48 \\
\hline 3,3-Dimethyl piperidine & $\mathrm{C}_{7} \mathrm{H}_{15} \mathrm{~N}$ & 113 & $113-84$ & 8.17 \\
\hline Butanoic acid, octylester & $\mathrm{C}_{12} \mathrm{H}_{24} \mathrm{O}_{2}$ & 200 & $112-71$ & 8.25 \\
\hline n-Butyric acid,2-ethylhexylester & $\mathrm{C}_{12} \mathrm{H}_{24} \mathrm{O}_{2}$ & 200 & $112-71$ & 8.25 \\
\hline Atropine & $\mathrm{C}_{17} \mathrm{H}_{23} \mathrm{NO}_{3}$ & 289 & $289-124$ & 14.43 \\
\hline Scopolamine & $\mathrm{C}_{17} \mathrm{H}_{23} \mathrm{NO}_{4}$ & 303 & $94-138$ & 15.46 \\
\hline Pyranone & $\mathrm{C}_{6} \mathrm{H}_{8} \mathrm{O}_{4}$ & 144 & $101-73$ & 4.25 \\
\hline Hyoscyamine & $\mathrm{C}_{17} \mathrm{H}_{23} \mathrm{NO}_{3}$ & 289 & $289-124$ & 14.43 \\
\hline Tropacocaine & $\mathrm{C}_{15} \mathrm{H}_{19} \mathrm{NO}_{2}$ & 245 & $124-82$ & 14.43 \\
\hline Tigloidine & $\mathrm{C}_{13} \mathrm{H}_{21} \mathrm{NO}_{2}$ & 223 & $124-82$ & 14.43 \\
\hline (Glycine,N-(aminoimino methyl)-N-methyl & $\mathrm{C}_{4} \mathrm{H}_{9} \mathrm{~N}_{3} \mathrm{O}_{2}$ & 131 & $113-84$ & 8.18 \\
\hline $\begin{array}{l}\text { Benzoicacid,3,5-dichloro-,8-aza-8-methyl }\{3,2,1\} \text { oct-3- } \\
\text { ylester }\end{array}$ & $\mathrm{C}_{15} \mathrm{H}_{17} \mathrm{Cl}_{2} \mathrm{NO}_{2}$ & 313 & $82-128$ & 14.39 \\
\hline Benzoyl ecgonine & $\mathrm{C}_{16} \mathrm{H}_{19} \mathrm{NO}_{4}$ & 289 & $124-289$ & 14.39 \\
\hline 8-Azabicyclo\{3,2,1 octane,3-chloro-8-methyl- & $\mathrm{C}_{8} \mathrm{H}_{14} \mathrm{ClN}$ & 159 & $82-124$ & 14.39 \\
\hline Benzoyl ecgonine & $\mathrm{C}_{16} \mathrm{H}_{19} \mathrm{NO}_{4}$ & 289 & $124-289$ & 14.39 \\
\hline
\end{tabular}

\subsection{Chemical compounds of aqueous crude extract of Datura innoxia leaves}

The chemical compounds identified in aqueous crude extract of Datura innoxia leaves are shown in Table 4 . Analysis by GC-MS revealed 31 compounds. 
Table 4 Chemical compounds of aqueous crude extract of Datura innoxia leaves

\begin{tabular}{|c|c|c|c|c|}
\hline Compound name & Formula & M. wt. & Base ions & RT \\
\hline d1-beta-phenyllaticacid.(dl-alpha-Hydroxyhydrocininnamic acid) & C9H10O3 & 166 & $91-106$ & 7.66 \\
\hline n-Hexadecanoic acid & $\mathrm{C} 16 \mathrm{H} 32 \mathrm{O} 2$ & 256 & $256-213$ & 12.03 \\
\hline 1,3-Dimethyl,-3,4,5,6-tetrahydro-2(1H)-rimidinone & C6H12N2O & 128 & $84-128$ & 6.88 \\
\hline 5-Aminovaleric acid & C5H11NO2 & 117 & $30-98$ & 3.46 \\
\hline Azlaic acid & $\mathrm{C} 9 \mathrm{H} 1604$ & 188 & $60-171$ & 7.40 \\
\hline 9,12-OctadecadienoicAcid (Z,Z),methylester & C19H3402 & 294 & $81-67$ & 13.20 \\
\hline 3-Methyladipicacid & C7H12O4 & 160 & $114-142$ & 7.05 \\
\hline Hyoscyamine & C17H23NO3 & 289 & $124-289$ & 13.29 \\
\hline Atropine & $\mathrm{C} 17 \mathrm{H} 23 \mathrm{NO} 3$ & 289 & $124-289$ & 13.92 \\
\hline Benzoicacid,3,5- dichloro,8-aza-8-methyl & $\mathrm{C} 15 \mathrm{H} 17 \mathrm{Cl} 2 \mathrm{~N}$ & 313 & $94-124$ & 14.54 \\
\hline Scopolamine & $\mathrm{C} 17 \mathrm{H} 21 \mathrm{NO} 4$ & 303 & $94-138$ & 15.54 \\
\hline Scopolamine, TMSderivative & $\mathrm{C} 20 \mathrm{H} 29 \mathrm{NO} 4$ & 375 & $94-138$ & 15.54 \\
\hline 1H-1midazole,1-acetyl & C5H6N2O & 110 & $68-129$ & 6.49 \\
\hline 5,6-dihydro-5-methyluracil & C5H8N2O2 & 128 & $56-138$ & 6.87 \\
\hline 9,12-Octadienoic acid(Z,Z) & $\mathrm{C} 18 \mathrm{H} 32 \mathrm{O} 2$ & 280 & $81-95$ & 10.92 \\
\hline 1,3-Dimethyl-3,4,5,6-tetrahyro-2(1H)-yridinone & $\mathrm{C} 6 \mathrm{H} 12 \mathrm{~N} 2 \mathrm{O}$ & 128 & $128-84$ & 6.87 \\
\hline Isocamphopinone & $\mathrm{C} 10 \mathrm{H} 160$ & 152 & $41-122$ & 3.84 \\
\hline Isovaleric acid, propyl ester & C8H16O2 & 144 & $103-85$ & 5.84 \\
\hline Butanoic acid,3-hydroxy-3-methyl- & C5H10O3 & 118 & $103-85$ & 5.84 \\
\hline Valeric acid,3-methyl & C6H12O2 & 116 & $87-60$ & 3.61 \\
\hline Aceticacid, phenyl & C8H8O2 & 136 & $91-136$ & 5.24 \\
\hline Oleic acid & C18H3402 & 282 & $282-82$ & 7.40 \\
\hline Azelaic acid & $\mathrm{C} 9 \mathrm{H} 1604$ & 188 & $111-83$ & 7.40 \\
\hline Tropacocaine & C15H19NO2 & 245 & $124-82$ & 14.43 \\
\hline Benzoyl ecgonine & C16H19N04 & 289 & $124-289$ & 14.39 \\
\hline 8-Azabicyclo $\{3,2,1\}$ octane,3-chloro- 8-methyl- & $\mathrm{C} 8 \mathrm{H} 14 \mathrm{ClN}$ & 159 & $82-124$ & 14.39 \\
\hline Apoatropin & C17H21NO2 & 271 & $94-124$ & 14.38 \\
\hline Homatropine & C16H21NO3 & 275 & $124-82$ & 14.37 \\
\hline 2-methylpyrazine-carboxylic acid & C6H6N2O2 & 138 & $138-94$ & 15.48 \\
\hline Pyrrolidine,1-(2-methylpropenyl)- & $\mathrm{C} 8 \mathrm{H} 15 \mathrm{~N}$ & 125 & $110-124$ & 14.39 \\
\hline 9,12-Octadecadieneylchloride, (Z,Z) & $\mathrm{C} 18 \mathrm{H} 31 \mathrm{ClO}$ & 298 & $81-95$ & 10.92 \\
\hline
\end{tabular}


Table 5 Mean weekly body weights of rats fed with methanol and aqueous extracts of Datura innoxia leaves and seeds for four weeks

\begin{tabular}{|l|l|l|l|l|l|}
\hline \multicolumn{1}{|c|}{ Groups } & \multicolumn{1}{c|}{ At zero } & \multicolumn{1}{c|}{$\mathbf{1}^{\text {st }}$ week } & \multicolumn{1}{c|}{ 2 $^{\text {nd }}$ week } & \multicolumn{1}{|c|}{$\mathbf{3}^{\text {rd }}$ week } & \multicolumn{1}{c|}{ (heek $^{\text {wh }}$} \\
\hline G 1 & \pm 113.00 & \pm 117.42 & \pm 121.10 & \pm 124.42 & \pm 128.34 \\
\hline G 2 & \pm 112.60 & \pm 114.14 & \pm 116.70 & \pm 118.96 & \pm 121.36 \\
\hline G 3 & \pm 113.60 & \pm 115.3 & \pm 117.58 & \pm 120.54 & \pm 123.68 \\
\hline G 4 & \pm 113.60 & \pm 115.38 & \pm 117.04 & \pm 119.14 & \pm 121.32 \\
\hline G 5 & \pm 113.40 & \pm 115.80 & \pm 118.00 & \pm 120.64 & \pm 123.78 \\
\hline G 6 & \pm 113.80 & \pm 113.64 & \pm 91.56 & \pm 69.94 & \pm 70.10 \\
\hline G 7 & \pm 113.80 & \pm 113.38 & \pm 68.08 & \pm 67.74 & \pm 67.10 \\
\hline G8 & \pm 113.80 & \pm 113.2 & \pm 90.38 & \pm 67.22 & \pm 44.80 \\
\hline G 9 & \pm 115.40 & \pm 114.94 & \pm 69.56 & \pm 69.28 & \pm 46.30 \\
\hline G 10 & \pm 114.20 & \pm 113.08 & \pm 68.38 & \pm 45.66 & \pm 22.70 \\
\hline G 11 & \pm 114.00 & \pm 113.04 & \pm 90.18 & \pm 22.62 & \pm 22.50 \\
\hline G12 & \pm 113.80 & \pm 112.98 & \pm 67.96 & \pm 22.4 & \pm 0.00 \\
\hline G13 & \pm 115.40 & \pm 114.16 & \pm 68.54 & \pm 22.62 & \pm 22.50 \\
\hline
\end{tabular}

*Values are expressed as means \pm STD (Standard deviation) for five rats in each Group for four weeks.

Group 1 (control), rats fed with normal rats' food and water. Group 2, 6 and 10 rats administered 40, 60 and 80mg/kg body weight aqueous seeds extracts respectively. Groups 4, 8 and 12 rats received 40,60 and 80mg/kg methanol seeds extracts respectively. Group 3, 7 and 11 rats received 40,60 and 80mg/kg body weight aqueous leaves extracts respectively. Group 5, 9 and 13 rats received 40,60 and 80mg/kg body weight methanol leaves extracts respectively.

Table 6 Comparative significant values of body weight

\begin{tabular}{|l|c|c|c|c|}
\hline Groups & 1st week & 2nd week & 3 $^{\text {rd }}$ week & 4 $^{\text {th }}$ week \\
\hline G1 & 0.111 & 0.119 & 0.011 & 0.024 \\
\hline G2 & 0.002 & 0.016 & 0.011 & 0.189 \\
\hline G3 & 0.001 & 0.019 & 0.050 & 0.075 \\
\hline G4 & 0.003 & 0.006 & 0.001 & 0.006 \\
\hline G5 & 0.007 & 0.014 & 0.020 & 0.040 \\
\hline G6 & 0.011 & 0.000 & 0.059 & 0.094 \\
\hline G7 & 0.000 & 0.071 & 0.074 & 0.162 \\
\hline G8 & 0.000 & 0.002 & 0.011 & 0.125 \\
\hline G9 & 0.011 & 0.096 & 0.122 & 0.000 \\
\hline G10 & 0.000 & 0.139 & 0.000 & 0.000 \\
\hline G11 & 0.000 & 0.001 & 0.000 & 0.000 \\
\hline G12 & 0.000 & 0.050 & 0.000 & 0.000 \\
\hline G13 & 0.002 & 0.011 & 0.000 & 0.000 \\
\hline
\end{tabular}




\subsection{Statistical analysis of Growth changes}

From the results of Tables ( 5 and 6 ) the data analysis by Analysis of variance (ANOVA) is a collection of statistical models and their associated estimation procedures (such as the "variation" among and between groups) used to analyze the differences among group means in a sample. ANOVA method showed that the means of body weight gain per week, increased in group 1 (control) from first week to the fourth week as below: (113.00, $\pm 117.42 \pm, 121.10 \pm, 124.42 \pm$ and $128.34 \pm$ ) respectively.

Also the method showed that the mean weekly body weight gain against daily oral doses of Datura innoxia seeds and leaves aqueous extracts for four weeks, for group 2 and group 3 at $40 \mathrm{mg} / \mathrm{kg} / \mathrm{body}$ weight dose were $(114.14 \pm, 116.70 \pm$, $118.96 \pm, 121.36 \pm, 115.30 \pm, 117.58 \pm, 120.54 \pm$ and 123.68 \pm ) respectively, there was an increase of body weight, but was lower than mean weekly body weight of control group, while at $40 \mathrm{mg} / \mathrm{kg}$ dose for group 4 and group 5 which received seeds and leaves methanol extracts for four weeks, their mean weekly body weight were (115.38 \pm , 117.04 \pm , $119.14 \pm, 121.32 \pm, 115.80 \pm, 118.00 \pm, 120.64 \pm$ and 123.78 \pm ) respectively, they were similar nearly to the mean of group 2 and group 3, thus there was an increase in body weight but also less than control. Group 6 and group 7 which were fed with $60 \mathrm{mg} \backslash \mathrm{kg}$ dose per day of aqueous seeds and leaves extracts, the mean of weekly body weight were as flow: $(113.64 \pm, 91.56 \pm, 69.94 \pm, 70.10 \pm, 113.38 \pm, 68.08 \pm, 67.74 \pm$ and $67.10 \pm)$ respectively, they had more lower mean of body weight than Group 1 ( control) and group 2 and group 3 which were fed with $40 \mathrm{mg} / \mathrm{kg}$ dose of aqueous seeds and leaves extracts, at the same time the mean weekly body weight of group 7 was less than group 6. Group 8 and group 9 which were fed with $60 \mathrm{mg} \backslash \mathrm{kg}$ dose per day of methanol seeds and leaves extracts, the mean of weekly body weight were as below: $(113.20 \pm, 90.38 \pm, 67.22 \pm, 44.80 \pm, 114.94 \pm, 69.56 \pm, 69.28 \pm$ and $46.3 \pm)$ respectively, this showed that the mean weekly body weight of $60 \mathrm{mg} / \mathrm{kg}$ dose of methanol seeds and leaves extracts was more less than $60 \mathrm{mg} / \mathrm{kg}$ dose of aqueous seeds and leaves extracts, so it revealed that the methanol seeds and leaves extracts had a high toxicity than aqueous seeds and leaves extracts. Group 10 and group 11 which were fed with $80 \mathrm{mg} \backslash \mathrm{kg}$ dose per day of aqueous seeds and leaves extracts, the mean of weekly body weight were as flow: $(113.08 \pm, 68.38 \pm, 45.66 \pm, 22.70 \pm, 113.04 \pm$, 90.18 \pm , $22.62 \pm$ and 22.50 \pm ) respectively, this declared that group 10 and group11 had most less mean of weekly body weight comparing to group ( 8 and 9) which received $60 \mathrm{mg} / \mathrm{kg}$ dose per day of aqueous seeds and leaves extracts and $60 \mathrm{mg} / \mathrm{kg}$ of methanol seeds and leaves extracts. Moreover, the mean weekly body weight of $80 \mathrm{mg} / \mathrm{kg}$ of aqueous leaves was less than the same dose of aqueous seeds extract, this indicated that the toxicity of aqueous leaves extract was higher than the aqueous seeds extract at the same dose. Group 12 and group 13 which were fed with 80 mg $\backslash \mathrm{kg}$ dose per day of methanol seeds and leaves extracts, the mean of weekly body weight were as flow: $(112.98 \pm, 67.96 \pm, 22.40 \pm, 0.00 \pm$, $114.16 \pm, 68.54 \pm, 22.62 \pm$ and $22.50 \pm$ ) respectively, this showed that the most low mean of weekly body weight in group (12 and 13) which were fed with $80 \mathrm{mg} / \mathrm{kg}$ dose per day of methanol seeds and leaves extracts comparing to all other groups. While the mean weekly of body weight for methanol seeds extract was higher than the methanol leaves at the same dose $(80 \mathrm{mg} / \mathrm{kg})$, this was great sign for a high toxicity of methanol leaves extract. According to above mention the mean weekly body weight of groups had lower $(\mathrm{p} \leq 0.05)$ body weight gains than control (Group 1). These results agree with the results of [14]in histopathological study of aqueous and methanolic extracts of Datura innoxia leaves and seeds on Wistar rats and the results of [15], in evaluation of analgesic effect of Datura stramonium leaves in hot plate and formalin test on male rats. The observation of body weight in groups (2, 3, 4 and 5) which treated with $40 \mathrm{mg} / \mathrm{kg}$ dose is agreement to the earlier report of [16] and [17] who have noted no change in body weight of rats treated with Datura Stramonium seeds with the exception of an increase in the relative lung weight.

\subsection{Effect of aqueous and methanolic extracts on histology of liver, brain and kidney}

The effects of methanolic and aqueous (leaves and seeds) extracts on experimental animals (rats) were mainly observed in three organs (kidney, liver and brain) for different orals doses which are (40,60and $80 \mathrm{mg} / \mathrm{kg} / \mathrm{day}$ ) for four weeks.

The histopathological changes observed in the organs were characteristic of some changes, this is in line with the observations made in the increasing or decreasing doses and the severity of the changes increased with increased of duration of the treatment of doses, suggesting a cumulative effect of the alkaloids and may due to the metabolites activities, these results agreed with [18] results in acute and chronic toxicity studies of Datura stranomnium stems in mice. In this study hepatocellular damage was observed especially at the doses (60 and 80) mg/kg body weight which indicates that there was a severe damage of liver, kidney and brain cells and tissues. Kidneys of rats that received 40 $\mathrm{mg} / \mathrm{kg} /$ day dose of methanol and aqueous (leaves and seeds) extracts, showed that there were: congestion, hemorrhage, oedema and swelling of cells and dilatation of renal tubules in the cortex and glomerular alteration (fatty cytoplasmic change) and segmentation of cells.Livers of rats that received $40 \mathrm{mg} / \mathrm{kg} / \mathrm{day}$ dose of methanolic and aqueous (leaves and seeds) extracts, showed that there were: congestion, oedema and hemorrhage of liver cells and centrilobular fibrosis. 
Brains of rats that received $(40 \mathrm{mg}) / \mathrm{kg} /$ day dose of methanol and aqueous (leaves and seeds) extracts, showed that there were: congestion, oedema and hemorrhage and hyperemic vessels in cerebellum.

Kidneys of rats that received $60 \mathrm{mg} / \mathrm{kg} /$ day dose of methanol and aqueous (leaves and seeds) extracts, showed that there were: glomerulonephritis, alteration (fatty change), hemorrahage, segmentation and degeneration of cells. Livers of rats that received $60 \mathrm{mg} / \mathrm{kg} /$ day dose of methanol and aqueous (leaves and seeds) extracts, showed there were: liver cells and tissues damage, centrilobular necrosis, hemorrhage and segmentation.Brains of rats that received $60 \mathrm{mg}$ $/ \mathrm{kg} /$ day dose of methanol and aqueous (leaves and seeds) extracts, showed that there were: cerebral neural congestion, cell degeneration, segmentation, oedema and packing in the cortex.Kidneys of rats that received $80 \mathrm{mg} / \mathrm{kg} / \mathrm{day}$ dose of methanolic and aqueous (leaves and seeds) extracts, showed that there were: tubular necrosis, inflammatory infiltration, severe hemorrhage in kidney tissue, damage of vessels and degenerated in cells and tissue.Livers of rats that received $80 \mathrm{mg} / \mathrm{kg} /$ day dose of methanolic and aqueous (leaves and seeds) extracts, showed that there were: Centrilobular necrosis, blood vessel dilatation, mild bleeding, necrosis and high segmentation and degeneration in cells and tissue. Brains of rats that received $80 \mathrm{mg} / \mathrm{kg} /$ day dose of methanolic and aqueous (leaves and seeds) extracts, showed that there were: hemorrhage, inflammatory cell, infiltration in brain, necrosis in brain tissue and degenerated neurons, cell and tissue of brain.

\section{Conclusion}

GC-MS analysis of Datura innoxia seeds and leaves (aqueous and methanol) extracts, revealed the presence of important pharmacological bioactive compounds mainly tropane alkaloids which are toxic at high concentrations, and fatty acids, phenyl propanoids, amino acids and flavonoids. The aqueous and methanol (seeds and leaves) extracts have nearly similar effect to the experimental rats, because they are all contain main alkaloidial compounds (atropine, hyoscyamine, tropacocaine, scopolamine, apoatropin) which have toxic effect. During the experiment loss in weight was noticed in the rats in group 6 up to group 13 may be due to reduction in intake of feed and this in turn may be attributed to the effect of the extracts administered to the animals. Histological study showed that extracts at doses $(40,60$ and 80 $\mathrm{mg} / \mathrm{kg} /$ day) caused pathologic changes. The effects were less marked in the low dose than the high dose Group. The lethal dose (LD50) of Datura innoxia seeds and leaves (methanol and aqueous) extracts in rats were nearly the same and parallel, that means they have the same efficacy due to their active ingredients (tropane alkaloids) this agree with the results of [19] in acute toxicity of aqueous and petroleum ether extracts of Daturainnoxia leaves in mice.

\section{Compliance with ethical standards}

\section{Acknowledgments}

Authors would like to thank the members and leaders of Forensic laboratories Sudan, especially who work in Forensic Chemistry for supporting and providing us with some information and technical analysis.

\section{Disclosure of conflict of interest}

The authors declare that there are no conflicts of interest.

\section{References}

[1] El-Astal, ZY, AERA Ashour and A. A. M. Kerrit. Antimicrobial activity of some medicinal plant extracts in Palestine. Pak. J Med. Sci., 2005; 21:187-193.

[2] Nascimento GF, Juliana L, Paulo CF and Giuliana LS. Antibacterial activity of plant extracts and phytochemicals on antibiotic-resistant bacteria. Brazilian Journal of Microbiology, 2008; 31(4): 247-256.

[3] Latif A, Ahmad H, Begum S, Adnan M, Hassian S, and Waseem, M. Medicinal and other economic plants as substitute to forest logging in Miandam and Sulatanr valleys, Swat. Proceedings of international workshop on conservation and sustainable use of medicinal and aromatic plants in Pakistan. WWF Pak., 2003; 101-105.

[4] United States Department of Agriculture- Agricultural Research Service (USDA-ARS, 2014). Germplasm Resources Information Network (GRIN). Online Database.

[5] United States Department of Agriculture- Natural Resources Conservation Service (USDA- (NRCS, 2014). The Plants Database. Baton Rouge, USA: National Plant Data Center, Online Database. Beltsville, Maryland, USA National Germplasm Resources Laboratory https://npgswebgrin. gov/gringlobal/taxon/taxonomysearch .aspx 
[6] AGIS. Agricultural Geo-Referenced Information System. 2007. http://www.agis.agric.za

[7] Schultes RE and Hofmann A. Plants of the gods. Healing Plants Press, New York. 1992; 192.

[8] Emboden W. Narcotic Plants alot of details about the history, chemistry and use of narcotic plants, including hallucinogens, stimulants, inebriants and hypnotics. 2008.

[9] Chopra RN, Nayar SL and Chopra IC. Glossary of Indian Medicinal Plants (Including the Supplement). Council of Scientific and Industrial Research, New Delhi. 1986;330.

[10] Weiner,N. Atropine, scopolamine and related antimuscarinic. 1985;76.

[11] Sukhdev. S H, Suman. P S K, Gennaro.L and Dev. D R. Extraction technologies for medicinal and aromatic plants. United Nation Industrial Development Organization and the International Center for Science and High Technology 2008; 116.

[12] Organization for Economic Cooperation and Development (OECD 425), Guide lines for the testing Chemicals acute oral toxicity- up-and down- procedure.2008.

[13] Anderw, HFK A Jacobson, J Rose and R Zeller. Hematoxylin and eosin staining of tissue and cell sections. Hematoxylin and eosin staining of tissue and cell sections. CSH Protoc., pdb.prot4986. 2008 DOI: 10.1101/pdb.prot4986

[14] Shama I Y, Y M Abd-kreem. andFadowa AA and Samar RM. Biochemical and Histopathological study of aqueous and methanolic extracts of Datura innoxia on Wistar rats. The Int. J. OF Advanced Research, 2014; 2(4): 878-887.

[15] KaliliNajabadim, and Atabis.M. Evaluation of analgesic effect of Datura stramonium leaves in hot plate and formalin test on male rats. Iranin Journal of Medicinal and Aromatic Plants, 2004; Vol. (20) No. (3):309-322.

[16] Gidado A, Zainab AA, Hadiza MU, Serah DP, Anas HY, Milala MA. Toxicity studies of ethanol extract of leaves of Daturastra moniumin rats. Afr. J. Biotechnol., 2007; 6: 1012-1015.

[17] Crawford L and Fredman M. The effects of low levels of dietary toxic weed seeds (jimson weed, Daturastra moniumand sicklepod, Cassia obtusifolia) on the relative size of rat liver and levels and function of cytochrome P450.Toxicol. Lett.,1990; 54:175-181.

[18] Udem SC, Obidoa O, Asuzu I U. Acute and chronic toxicity studies of Datura stramonium stem bark extract in mice. Comp. Clin. Pathol. ,2009; 19:275-282.

[19] M.A. Abo Kutaifa, D. A. Abbas and FMkadhim. Acute toxicity of aqueous and petroleum ether extracts of Datura innoxia leaves in mice. College of Veterinary Medicine University of Baghdad, Al-Anbar J.Vet.Sci., 2012; Vol. (5) No. (2): 68-74. 